OPEN ACCESS

Edited by:

Oliver Diaz,

University of Barcelona, Spain

Reviewed by:

Huaiqiang Sun,

Sichuan University, China

Wenwu Ling,

Sichuan University, China

*Correspondence:

Jun Gong Zhao

zhaojungongradio@hotmail.com

Specialty section:

This article was submitted to

Cancer Imaging and

Image-directed Interventions,

a section of the journal

Frontiers in Oncology

Received: 20 April 2021 Accepted: 11 August 2021 Published: 09 September 2021

Citation:

Hu L, Zhou DW, Fu CX, Benkert T, Xiao YF, Wei LM and Zhao JG (2021)

Calculation of Apparent Diffusion

Coefficients in Prostate Cancer Using Deep Learning Algorithms: A Pilot Study.

Front. Oncol. 11:697721.

doi: 10.3389/fonc.2021.697721

\section{Calculation of Apparent Diffusion Coefficients in Prostate Cancer Using Deep Learning Algorithms: A Pilot Study}

\author{
Lei Hu ${ }^{1}$, Da Wei Zhou ${ }^{2}$, Cai Xia Fu ${ }^{3}$, Thomas Benkert ${ }^{4}$, Yun Feng Xiao ${ }^{1}$, Li Ming Wei ${ }^{1}$ \\ and Jun Gong Zhao ${ }^{1 *}$ \\ ${ }^{1}$ Department of Diagnostic and Interventional Radiology, Shanghai Jiao Tong University Affiliated Sixth People's Hospital, \\ Shanghai, China, 2 State Key Laboratory of Integrated Services Networks, School of Telecommunications Engineering, Xidian \\ University, Xi'an, China, ${ }^{3}$ Magnetic Resonance (MR) Application Development, Siemens Shenzhen Magnetic Resonance Ltd., \\ Shenzhen, China, ${ }^{4}$ MR Application Predevelopment, Siemens Healthcare GmbH, Erlangen, Germany
}

Background: Apparent diffusion coefficients (ADCs) obtained with diffusion-weighted imaging (DWI) are highly valuable for the detection and staging of prostate cancer and for assessing the response to treatment. However, DWI suffers from significant anatomic distortions and susceptibility artifacts, resulting in reduced accuracy and reproducibility of the ADC calculations. The current methods for improving the DWI quality are heavily dependent on software, hardware, and additional scan time. Therefore, their clinical application is limited. An accelerated ADC generation method that maintains calculation accuracy and repeatability without heavy dependence on magnetic resonance imaging scanners is of great clinical value.

Objectives: We aimed to establish and evaluate a supervised learning framework for synthesizing ADC images using generative adversarial networks.

Methods: This prospective study included 200 patients with suspected prostate cancer (training set: 150 patients; test set \#1: 50 patients) and 10 healthy volunteers (test set \#2) who underwent both full field-of-view (FOV) diffusion-weighted imaging ( $f$-DWI) and zoomed-FOV DWI (z-DWI) with $b$-values of $50,1,000$, and $1,500 \mathrm{~s} / \mathrm{mm}^{2}$. ADC values based on f-DWI and z-DWI (f-ADC and z-ADC) were calculated. Herein we propose an $A D C$ synthesis method based on generative adversarial networks that uses $\mathrm{f}-\mathrm{DWI}$ with a single $b$-value to generate synthesized ADC (s-ADC) values using z-ADC as a reference. The image quality of the s-ADC sets was evaluated using the peak signal-to-noise ratio (PSNR), root mean squared error (RMSE), structural similarity (SSIM), and feature similarity (FSIM). The distortions of each ADC set were evaluated using the T2-weighted image reference. The calculation reproducibility of the different ADC sets was compared using the intraclass correlation coefficient. The tumor detection and classification abilities of each ADC set were evaluated using a receiver operating characteristic curve analysis and a Spearman correlation coefficient. 
Results: The s-ADC $\mathrm{b}_{\mathrm{b} 1000}$ had a significantly lower RMSE score and higher PSNR, SSIM,

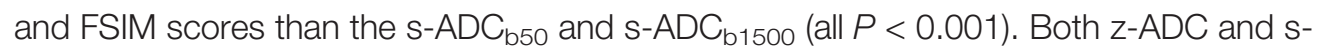
$A D C_{b 1000}$ had less distortion and better quantitative $A D C$ value reproducibility for all the evaluated tissues, and they demonstrated better tumor detection and classification performance than f-ADC.

Conclusion: The deep learning algorithm might be a feasible method for generating ADC maps, as an alternative to z-ADC maps, without depending on hardware systems and additional scan time requirements.

Keywords: apparent diffusion coefficient, diffusion magnetic resonance imaging, deep learning, prostatic neoplasms, supervised machine learning

\section{INTRODUCTION}

Diffusion-weighted imaging (DWI) currently constitutes an integral part of multiparametric magnetic resonance imaging (MRI) examinations of the prostate. Apparent diffusion coefficients (ADCs) obtained with DWI are highly valuable for detecting and staging prostate cancer, evaluating cancer aggressiveness $(1,2)$, guiding targeted biopsies, and assessing the response to treatment (3-10). Clinically, the accuracy of the ADC measurement depends on the quality of the DWI image.

Single-shot echo-planar imaging (SS-EPI)-based sequences are preferred for DWI because of its ability to acquire the images rapidly and the robustness of the technique against motion artifacts. However, because of its high sensitivity to chemical shifts and magnetic susceptibilities (11), conventional SS-EPI DWI suffers from significant anatomic distortions (12) and susceptibility artifacts, resulting in reduced ADC calculation accuracy and reproducibility (12-14). Another limitation is the low signal-tonoise ratios observed during DWI, which result in noise-induced signal intensity biases $(15,16)$ and inaccurate $\mathrm{ADC}$ maps. These drawbacks may lead to an error in judgment regarding the condition of a patient and a potential misdiagnosis of malignant lesions or over-treatment of benign lesions. Zoomed field-of-view (FOV) DWI (z-DWI) is an appealing attempt to address these limitations. This method reduces the scanning time as well as artifacts, distortions, and blurring of images, and it also has improved spatial resolution $(17,18)$. Additionally, z-DWI can effectively improve the ADC map accuracy $(17,18)$; however, the technique depends on radio frequency design and software platforms (17-19), which can make it unaffordable for many small- and medium-sized hospitals and their patients. Moreover, a reduced FOV may prevent the visualization of lymph nodes (3). Therefore, the clinical application of $z$-DWI is limited. A method that can consistently generate high-quality ADC images with reduced equipment costs will be of more benefit to patients in clinical practice.

Recently, the advent of generative adversarial networks (GANs) (20) has shown promise for optimizing medical image quality without relying on software and equipment conditions (21). As a generative model, the objective of a GAN is to learn the underlying training data distributions to generate realistic images that are indistinguishable from the input datasets (21). With their ability to mimic data distributions, GANs have been used to translate lowquality images into high-quality counterparts. Previous studies have successfully used GANs to improve computed tomography (CT) or MRI quality in terms of de-noising (22), increased resolution (23), artifact reduction (24), and motion correction (25). Inspired by these image optimization solutions, we hypothesized that deep learning algorithms based on GANs might be promising for generating ADC maps with good image quality and improved ADC calculation accuracy. The purpose of this study was to establish and evaluate a supervised learning framework based on a GAN to synthesize realistic zoomed FOV ADC images using conventional full FOV SS-EPI DWI images with a single $b$-value.

\section{MATERIALS AND METHODS}

\section{Patients and Healthy Volunteers}

This prospective study was approved by the local ethics committee, and informed consent was obtained from each participant. All the procedures involving human participants were performed in accordance with the 1964 Helsinki Declaration and its later amendments. A total of 200 consecutive patients underwent preoperative MRI examinations and subsequent MRI fusion ultrasound-guided biopsies for suspected prostate cancer (PCa) between December 2018 and May 2020. The inclusion criteria were as follows: patients with (1) at least one prostate lesion visible on DWI and ADC maps and (2) complete clinical information and pathologic examination information, including biopsy reports. Ten healthy volunteers were also recruited for the study. The study included four steps: (1) MRI examinations, (2) model training, (3) image quality assessments, and (4) ADC assessments (Figure 1).

\section{MRI Examinations and Datasets}

All the patients and volunteers underwent multiparametric MRI examinations of the prostate using a 3T MRI scanner (MAGNETOM Skyra, Siemens Healthcare, Erlangen, Germany) equipped with a phased-array 18-channel body coil and an integrated 32-channel spine coil. Both a transversal single-shot full FOV-EPI DWI (f-DWI) and a prototypic non-parallel transmission zoomed EPI DWI (z-DWI) with $b$-values of 50 , 1,000 , and $1,500 \mathrm{~s} / \mathrm{mm}^{2}$ were performed with the ADC reconstruction maps ( $\mathrm{f}-\mathrm{ADC}$ and $\mathrm{z}$-ADC) using a standard 


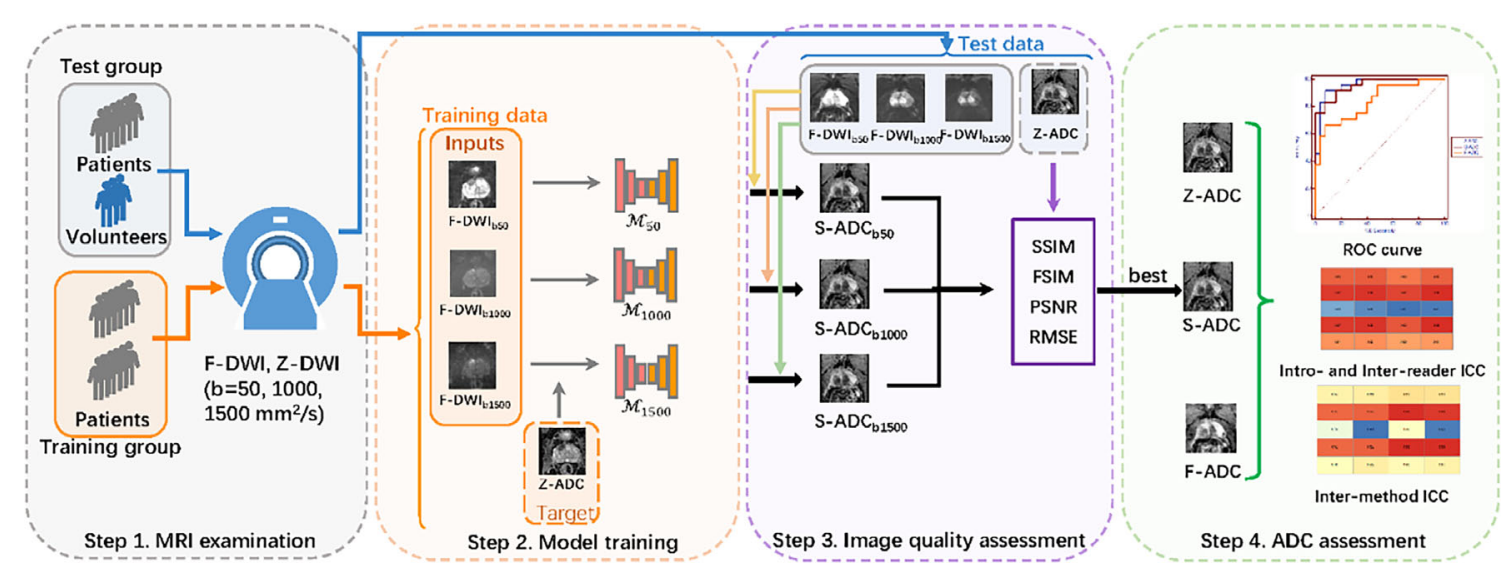

FIGURE 1 | Overall study flow diagram. Step 1: All the patients and healthy volunteers underwent multiparametric magnetic resonance imaging examinations of the prostate, including full field-of-view (FOV) diffusion-weighted imaging (f-DWI) and zoomed FOV diffusion-weighted imaging with $b$-values of $50,1,000$, and 1,500 s/mm². Step 2: The models that used full f-DWl with different $b$-values ( $f-D W l_{b 50}, f-D W l_{b 1000}$, and f-DWl $\left.\right|_{b 1500}$ ) to synthesize the apparent diffusion coefficient (s-ADC) maps (s$A D C_{b 50}, s-A D C_{b 1000}$, and s-ADC $\left.C_{b 1500}\right)$ were trained. Step 3: The image quality of s-ADC $C_{b 50}, s-A D C_{b 1000}$, and s-ADC $C_{b 1500}$ were evaluated using the peak signal-tonoise ratio, root mean square error, structural similarity, and feature similarity. Step 4: An ADC assessment was performed to determine reproducibility, tumor detection, and classification.

mono-exponential with all the acquired $b$-values (14). Axial T2weighted images were obtained from all the participants, and the total examination time was approximately $7 \mathrm{~min}$ and $40 \mathrm{~s}$. The detailed scan parameters are shown in Table $\mathbf{1}$.

Patient images were randomly divided into two groups (training set: 150 patients, test set \#1: 50 patients). The training set was used to build the framework and train different models to synthesize the ADC maps (s-ADCs). Test set \#1 was used to test the reproducibility of the s-ADC prostate lesion measurements, along with tumor detection. The images of the healthy volunteers were regarded as test set \#2, which was used to test the reproducibility and consistency of the normal prostate tissue s$\mathrm{ADC}$ calculations, including the peripheral zone (PZ) and the transitional zone (TZ).

\section{Data Pre-Processing}

Before the model training could occur, image selection, cropping, and registration were performed on $\mathrm{f}$-DWI with $b$-values of 50 , 1,000 , and $1,500 \mathrm{~s} / \mathrm{mm}^{2}$ and the $\mathrm{z}$-ADC images. The first and last slices that did not cover the prostate were removed manually. The images with severe distortion and artifacts were also removed. Ultimately, there were between five and 20 DWI images selected for each person. Finally, there were 2,250 images from each set for the 150 patients in the training set, 750 images from each set for the 50 patients in test set \#1, and 145 images from each set for the 10 healthy volunteers in test set \#2.

Due to hardware limitations of the graphics cards and the CPU memory, we used only axial slices of the cropped data to train the two-dimensional generation models. The f-DWI data had an original voxel size of $2.13 \times 2.13 \times 3.3 \mathrm{~mm}^{3}$ and a matrix size of $178 \times 132$, whereas the $\mathrm{z}$-ADC data had a voxel size of $0.95 \times 0.95 \times 3.3 \mathrm{~mm}^{3}$ and a matrix size of $112 \times 200$. The f-DWI data were first resampled to a voxel size of $0.95 \times 0.95 \times 3.3 \mathrm{~mm}^{3}$ with a matrix size of $360 \times 267$, and both modalities were cropped at the center to extract the relevant prostate region. The f-DWI data were then aligned to the z-ADC data using the affine transformation implemented by the Advanced

TABLE 1 | The magnetic resonance imaging sequence parameters.

\begin{tabular}{|c|c|c|c|}
\hline Parameter & T2-weighted imaging & F-DWI & Z-DWI \\
\hline Field-of-view, FOV $\left(\mathrm{mm}^{2}\right)$ & $200 \times 200$ & $380 \times 280$ & $190 \times 106$ \\
\hline Imaging matrix & $320 \times 320$ & $132 \times 178$ & $112 \times 200$ \\
\hline Thickness (mm) & 3.5 & 3 & 3 \\
\hline Distance fact & 0 & $10 \%$ & $10 \%$ \\
\hline B-value $\left(\mathrm{s} / \mathrm{mm}^{2}\right)$ & n.a. & $50,1,000,1500$ & $50,1,000,1500$ \\
\hline Echo time (ms) & 101 & 73 & 76 \\
\hline Time to repeat (ms) & 6,000 & 4,200 & 3,800 \\
\hline Bandwidth (Hz/pixel) & 200 & 1,872 & 1,612 \\
\hline Scan time (min) & $2: 08$ & 3:05 & $2: 27$ \\
\hline
\end{tabular}

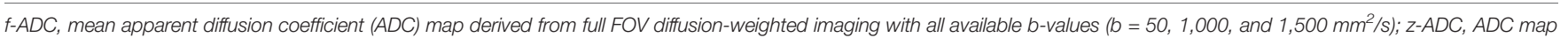
derived from zoomed FOV diffusion-weighted imaging with all available b-values ( $b=50,1,000$, and 1,500 $\left.\mathrm{mm}^{2} / \mathrm{s}\right)$, n.a., no available. 
Normalization Tools (https:/github.com/ANTsX/ANTs). To facilitate the model training, all the two-dimensional axial slices were scaled to a unified resolution of $224 \times 224$ pixels.

To select a suitable $b$-value for ADC synthesis, we first used 2,250 paired f-DWI images with $b$-values of $50 \mathrm{~s} / \mathrm{mm}^{2}$ and the ground truth $\mathrm{z}$-ADC maps from the training set as inputs and references, respectively, to train our framework-based model $\mathrm{M}_{50}$ to synthesize ADC maps (s-ADC ${ }_{b 50}$ ). Similarly, the $M_{1000}$ and $M_{1500}$ models based on the f-DWI images with $b$-values of 1,000 and 1,500 $\mathrm{s} / \mathrm{mm}^{2}$ were trained to synthesize ADC maps (s-ADC $\mathrm{b}_{1000}$ and $\left.\mathrm{s}-\mathrm{ADC}_{\mathrm{b} 1500}\right)$.

\section{Model Training}

We have proposed a GAN-based framework to generate realistic z-ADC maps from f-DWI maps (Figure 2).

The generator $G$ translates the input image (f-DWI) $X$ into the synthesized ADC (s-ADC) $S$ with a quality similar to the ground truth $\mathrm{z}$-ADC, which is used as reference $Y$. The discriminator $D$ takes either the $S$ or the $Y$ as input and determines whether the input is a real z-ADC map. Specifically, we used a deep residual network structure with skip connections to construct the generator $G$ and a full convolution network to construct the discriminator $D$. The adversarial loss of the discriminator $D$ is formulated as follows:

$$
\mathcal{L}_{D}=\mathbb{E}_{Y \sim P_{Y}}\left[(D(Y)-1)^{2}\right]+E_{S \sim P_{S}}\left[(D(S))^{2}\right]
$$

The adversarial loss of the generator $G$ is formulated as follows:

$$
\mathcal{L}_{G}^{a d v}=\mathbb{E}_{S \sim P_{S}}\left[(D(S)-1)^{2}\right]
$$

Considering that the standard GAN might not adequately preserve the tumors/lesions during image-to-image translation (26), we introduced a multi-level verification (MLV) mechanism, including a pre-trained recognition model $C$. This mechanism promotes the generator $G$ to better retain the features, which helps in the diagnosis. Using $C$, the proposed MLV mechanism provides more details about the tumor/lesion features when they are extracted from the input images. $G$ represents the tumor/ lesion texture, making it better and more robust against changes in appearance and geometric transformations (27).

We first obtained a recognition model $C$, which was pretrained on a VGG-19-based network using the processed images from the patients and healthy volunteers with a benign or malignant label (28). Subsequently, the multiple layers of model $C$ extracted the multi-level features from the fake synthetic ADC map $S$ and the ground truth ADC map. The sum of the mean square errors of the features in each level layer was used as the multi-level feature loss to supervise the generator $G$.

Inspired by the current work (29) and considering the use of multi-level features, we selected the features in the $0,1 \mathrm{st}, 3 \mathrm{rd}$, and 5 th level layers. The loss of the multi-level verification mechanism is formulated as follows:

$$
\mathcal{L}_{G}^{m l v}=\Sigma_{i=0,1,3,5} \theta_{i} \cdot\left\|C^{i}(S)-C^{i}(Y)\right\|_{2}^{2}
$$

where $\theta_{i} \in(0,1)$ denotes the weight parameter for the loss $\left(\mathcal{L}_{\text {mlf }}^{i}\right)$ at different levels, and it is optimized in each epoch to cause a faster decrease in the loss of the larger items. The $\theta_{i}$ in the $j$ - th epoch can be computed as follows:

$$
\theta_{i}^{j}=\Sigma_{n=0,1,3,5} \frac{\left\|C_{i}^{j-1}(S)-C_{i}^{j-1}(Y)\right\|_{2}^{2}}{\left\|C_{n}^{j-1}(S)-C_{n}^{j-1}(Y)\right\|_{2}^{2}}
$$

where $C_{i}^{j-1}(\cdot)$ denotes the feature of the $i$ - th layer in the $(j-1)$-th epoch, and $C_{n}^{j-1}(\cdot)$ indicates the feature of the $n$ - th layer in the $(j-1)$-th epoch. We initialized $\theta_{i}$ to $1 / 4$. The objective function of generator $G$ is formulated as follows:

$$
\mathcal{L}_{G}=\mathcal{L}_{G}^{a d v}+\lambda_{1} \mathcal{L}_{G}^{m l v}
$$

with $\gamma_{1}$ set to $10^{-1}$.

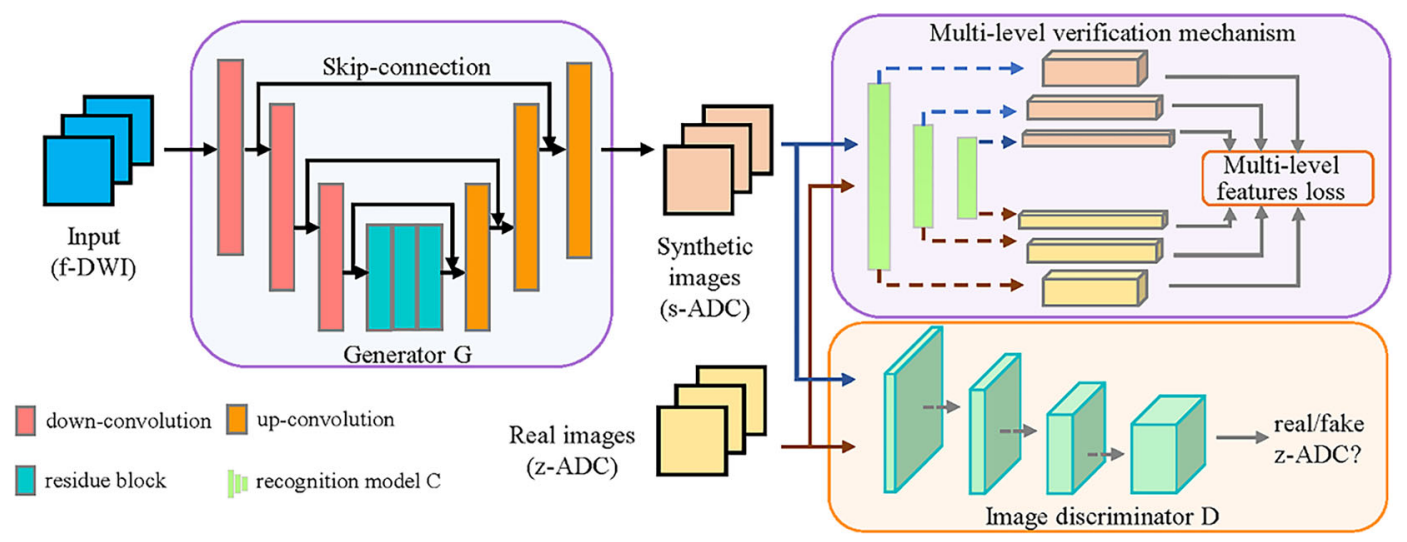

FIGURE 2 | Illustration of our framework. The proposed framework consists of a generator (G), which was constructed using a deep convolution network with skip connections, and an image discriminator $(D)$ constructed using a full convolution network. The $G$ transforms the f-DWl into a synthesized apparent diffusion coefficient (s-ADC) using zoomed field-of-view diffusion-weighted imaging (z-ADC) as a reference. The $D$ takes either s-ADC or z-ADC as the input and determines whether the input is a real z-ADC. In addition, to promote $G$ in an effort to retain better features for diagnosis, we introduced a multi-level verification mechanism, including a pre-trained recognition model $(C)$, to extract the multi-level features from the s-ADC and the z-ADC. 


\section{Experimental Settings}

The generator consists of three convolution layers, followed by five residual blocks and three deconvolution layers. Each convolution or deconvolution layer is followed by an instancenormalization layer and a ReLu activation layer. The discriminator consists of five convolution layers. The learning rate was set to 0.001 for both the generator and the discriminator. The batch size was set to 5 , and the epoch was set to 50 . The details of the generator and discriminator can be found at https:// github.com/huxiaolie/ADC_generation. All the algorithms were implemented using Python 3.6 (https://www.python.org/ downloads/release/python-362/) and Pytorch 1.6.0 (https:// pytorch.org/get-started/previous-versions/) on an Ubuntu 16.04 system with an NVIDIA TITAN XP GPU.

\section{Image Quality Assessment}

The s-ADC sets were synthesized using each model with inputs from the $\mathrm{f}$-DWI images with $b$-values of 50, 1,000, and 1,500 s/ $\mathrm{mm}^{2}$ for test set \#1 (50 patients) and test set \#2 (10 healthy volunteers), and they were compared using peak signal-to-noise ratios (PSNRs), root mean square errors (RMSEs), structural similarities (SSIMs), and feature similarities (FSIMs) (30).

A radiologist with 6 years of experience with prostate MRIs measured the anterior-posterior (AP) and left-right (LR) diameters of each prostate on the ADC set on the slice on which the prostate showed the greatest cross-sectional area. The differences in the measured AP and LR diameters of the prostate relative to the T2-weighted image (T2WI) were computed for $\mathrm{f}$ $\mathrm{ADC}, \mathrm{z}-\mathrm{ADC}$, and s-ADC, with the best performance from the above-mentioned quantitative evaluation.

\section{ADC Measurement Assessment}

For the patient study, two radiologists with 5 and 10 years of experience with prostate MRIs and who were unaware of the clinical, surgical, and histologic findings independently drew a circular region of interest (ROI) with an area of approximately $0.5-0.8 \mathrm{~cm}^{2}$ in the center of the lesion, excluding its edges. For the healthy volunteer study, the readers drew circular ROIs with an area of approximately $0.5 \mathrm{~cm}^{2}$ in the peripheral and transitional zones on the ADC maps using axial T2-weighted images as the anatomical reference. The mean $\mathrm{ADC}$ values for each ROI were recorded.

The ADC sets of all the patients and healthy volunteers were measured twice using Image J (NIH Image, Bethesda, MD) in a different order, with an interval of 2 weeks. The first measurement given by the two readers showed the consistency of the ADC measurements for each ADC set. The second measurement showed the repeatability of the ADC values for each ADC set.

\section{Tumor Detection Assessment}

The s-ADC set with the best image quality and ADC measurement assessment among the three s-ADC sets was selected for tumor detection assessments. The selected s-ADC was compared with the f-ADC and z-ADC in terms of the ability to differentiate benign from malignant lesions. The correlation between the $\mathrm{ADC}$ values in the different $\mathrm{ADC}$ sets and tumor grades was also evaluated.

\section{Statistical Analyses}

Analyses of the baseline characteristics between the training group and the test group were conducted. An independent $t$ test was used to assess normally distributed continuous variables. The Mann-Whitney $U$-test was used to assess non-normally distributed continuous variables.

To assess differences in the image quality metrics (PSNR, RMSE, SSIM, and FSIM) between any two s-ADC sets, a paired Student's $t$-test was applied. The intraclass correlation coefficient (ICC) was used to assess the inter-and intra-reader repeatability of the ADC measurements for each tissue (malignant lesion, benign lesion, peripheral zone, and transitional zone) in each $\mathrm{ADC}$ set (f-ADC, $\mathrm{z}-\mathrm{ADC}$, and s-ADC). The ICC was also used to evaluate the inter-method reliability of the ADC values for each tissue between the synthesized image (s-ADC) and the reference image ( $\mathrm{z}-\mathrm{ADC}$ ). A receiver operating characteristic (ROC) curve analysis was performed to assess the ability to discriminate between benign and malignant prostate lesions based on the $\mathrm{ADC}$ values. The differences in the area under the curve (AUC) values were tested using DeLong tests. The statistical analyses were performed using MedCalc software. Two-tailed tests were used to calculate all the $P$-values. Statistical significance was set at $P<0.05$.

\section{RESULTS}

\section{Demographic Characteristics}

The patient characteristics are summarized in Table 2. There were no significant differences in the mean ages between the patients with and without PCa $(P=0.557)$. The mean prostatespecific antigen (PSA) level was significantly higher in patients with PCa compared to those without PCa $(P<0.001)$.

There were no significant differences in mean ages and mean PSA between the training set and test set \#1 (mean ages: $68 \pm 10$ vs. $68 \pm 12$ years, $P=0.974$; PSA: $29.872 \pm 69.461$ vs. $39.296 \pm$ $92.604, P=0.154$ ). The mean age of test set \#2 (healthy volunteers, $24 \pm 3$ years) is significantly lower than that of the training set and test set $\# 1(P<0.001)$.

\section{Image Quality Assessment}

Visual comparisons of the s-ADC values generated with different $b$-value inputs are shown in Figure 3. We observed that the s$\mathrm{ADC}_{\mathrm{b} 50}$ displayed blurred images of the prostate, bladder, rectum, pelvic floor muscles, and pubic symphysis in both the patients and the volunteers. Compared with s-ADC $\mathrm{b}_{\mathrm{b} 0}$, s$\mathrm{ADC}_{\mathrm{b} 1000}$ and $\mathrm{s}-\mathrm{ADC}_{\mathrm{b} 1500}$ could delineate normal tissues and lesions more clearly and sharply, which was in line with the 
TABLE 2 | The clinical characteristics of the patient cohort.

\begin{tabular}{|c|c|c|c|}
\hline Characteristics & Patients without cancer $(n=106)$ & Patients with cancer $(n=94)$ & $P$-value \\
\hline Mean age (y) [range] & $70(52-87)$ & 71 (48-88) & 0.675 \\
\hline total PSA (ng/ml) & $11.079 \pm 9.013$ & $57.002 \pm 125.88$ & $<0.001$ \\
\hline \multicolumn{4}{|l|}{ Position, no. } \\
\hline Peripheral zone & 44 & 63 & $<0.001$ \\
\hline Transitional zone & 62 & 31 & \\
\hline \multicolumn{4}{|l|}{ Gleason score (n, \%) } \\
\hline 6 & -- & 8 & \\
\hline 7 & -- & 46 & \\
\hline 8 & -- & 24 & \\
\hline 9 & -- & 16 & \\
\hline
\end{tabular}

The data are mean \pm standard deviation, unless otherwise indicated.

PSA, prostate-specific antigen.

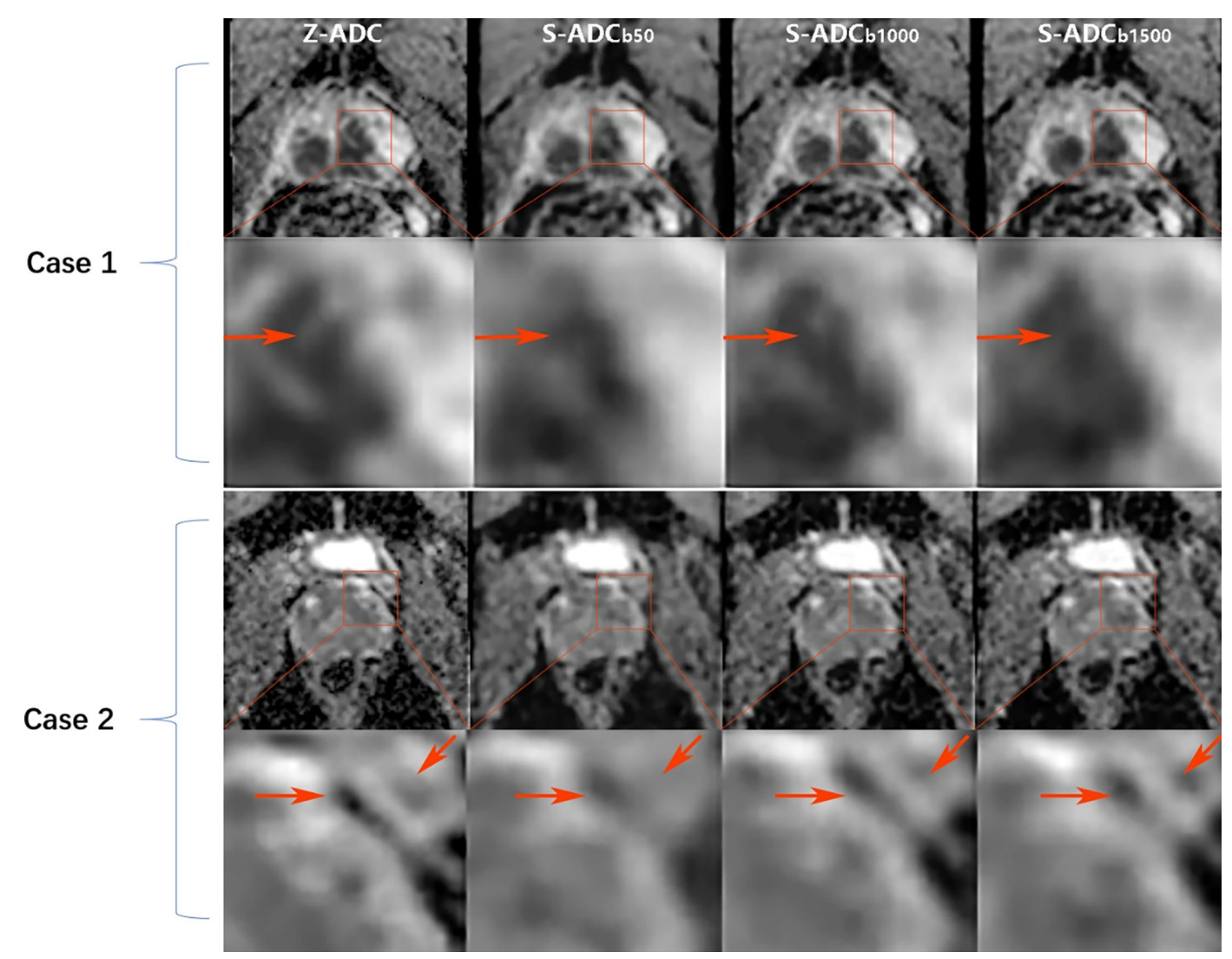

FIGURE 3 | Comparison of the synthesized apparent diffusion coefficient (s-ADC) maps. Case 1: An 82-year-old man with prostate cancer from test set 1 who had an initial prostate-specific antigen level of $13.04 \mathrm{ng} / \mathrm{ml}$. Case 2: A 27 -year-old healthy man from test set 2. For these two cases, both the s-ADC $\mathrm{b}_{\mathrm{b} 1000}$ and $s-A D C_{b 1500}$ performed well in displaying the prostate, pelvic floor muscles, pubic symphysis, and the entire cancer lesion. However, the $s-A D C_{b 50}$ images of these structures are fuzzy. According to the local enlargement of the images (the images in the second and fourth lines), the s-ADC to the $\mathrm{s}-\mathrm{ADC}_{\mathrm{b} 1500}$, and it retains more details of the $\mathrm{z}-\mathrm{ADC}$ (shown as red arrows).

ground truth. According to the magnified images of the local tissue structures, $\mathrm{s}-\mathrm{ADC}_{\mathrm{b} 1000}$ provided more details than s$\mathrm{ADC}_{\mathrm{b} 1500}$ with reference to $\mathrm{z}$-ADC.

As shown in the violin plots (Figure 4), s- $\mathrm{ADC}_{\mathrm{b} 1000}$ performed better than the other two s-ADC sets in terms of the distribution, median, and inter-quartile ranges of the
RMSE, SSIM, FSIM, and PSNR scores. The mean RMSE scores of $\mathrm{s}-\mathrm{ADC}_{\mathrm{b} 50}, \mathrm{~s}-\mathrm{ADC}_{\mathrm{b} 1000}$, and $\mathrm{s}-\mathrm{ADC}_{\mathrm{b} 1500}$ were $4.1 \times$ $10^{-3}, 2.5 \times 10^{-3}$, and $3.1 \times 10^{-3}$, respectively. The mean PSNR scores of $\mathrm{s}-\mathrm{ADC}_{\mathrm{b} 50}, \mathrm{~s}-\mathrm{ADC}_{\mathrm{b} 1000}$, and $\mathrm{s}-\mathrm{ADC}_{\mathrm{b} 1500}$ were 48.0 , 53.4 , and 51.0, respectively. The mean SSIM scores of s$\mathrm{ADC}_{\mathrm{b} 50}, \mathrm{~s}-\mathrm{ADC}_{\mathrm{b} 1000}$, and $\mathrm{s}-\mathrm{ADC}_{\mathrm{b} 1500}$ were $0.972,0.986$, and 

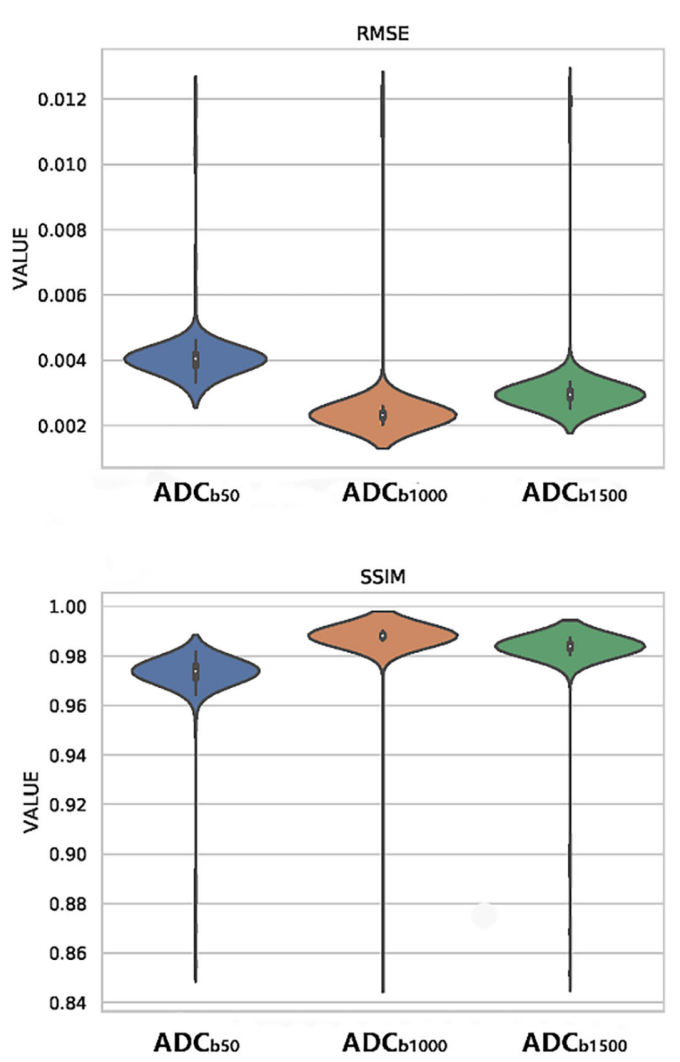

FIGURE 4 | Violin plots of the quantitative metric distributions of the s-ADC sets.
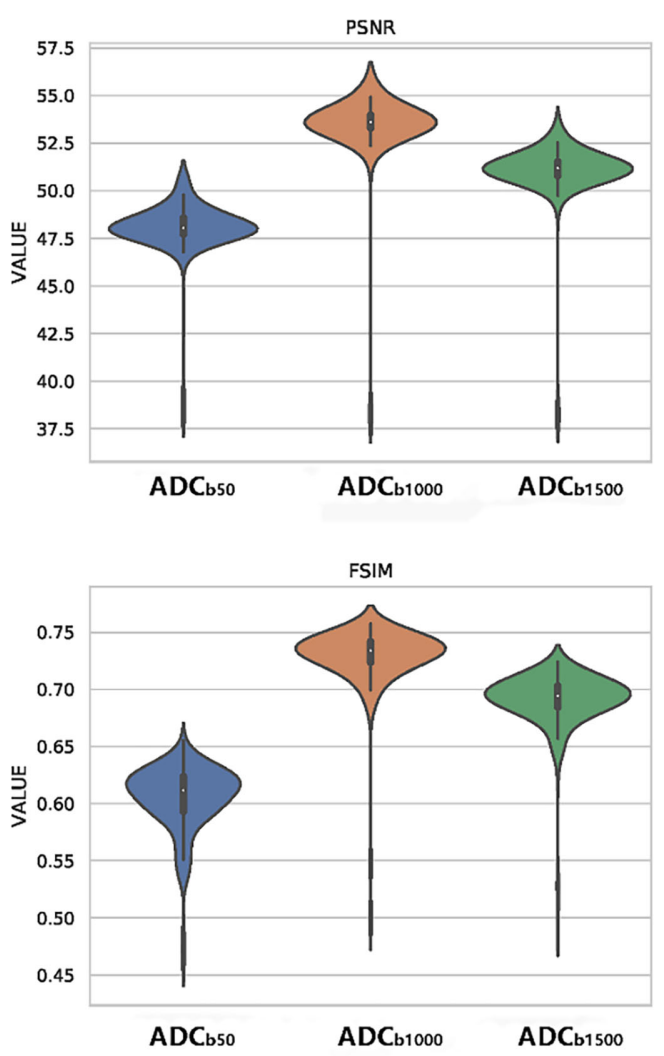

0.982, respectively. The mean FSIM scores of $\mathrm{s}-\mathrm{ADC}_{\mathrm{b} 50}$. s$\mathrm{ADC}_{\mathrm{b} 1000}$, and $\mathrm{s}-\mathrm{ADC}_{\mathrm{b} 1500}$ were $0.604,0.728$, and 0.690 , respectively. $\mathrm{s}-\mathrm{ADC}_{\mathrm{b} 1000}$ had a significantly lower RMSE score and higher PSNR, SSIM, and FSIM scores than s$\mathrm{ADC}_{\mathrm{b} 50}$ and $\mathrm{s}-\mathrm{ADC}_{\mathrm{b} 1500}($ all $P<0.05)$.

To evaluate the distortion in the ADC maps, the differences in the AP and LR diameters of the prostate relative to T2WI were both significantly lower for s-ADC (AP, 2.734; LR, 3.204) and z-ADC (AP, 2.755; LR, 3.073) than for f-DWI (AP, 5.916; LR, 5.053) (all $P<0.001)$.

\section{ADC Measurement Assessment}

The measurements of all the ADC sets (f-ADC, z-ADC, $\mathrm{s}-\mathrm{ADC}_{\mathrm{b} 50}, \mathrm{ADC}_{\mathrm{b} 1000}$, and $\mathrm{s}-\mathrm{ADC}_{\mathrm{b} 1500}$ ) on various tissues from both readers are presented in Table 3. For all the ADC sets, the $\mathrm{ADC}$ values of the $\mathrm{TZ}$ are significantly lower than those of the $\mathrm{PZ}$, while the ADC values of the malignant lesions are significantly lower than those of the benign lesions (all $P<0.05$ ).

Figure 5 presents the results of the intra-reader reproducibility (Figures 5A, B) and inter-reader consistency (Figure 5C) analyses for each $\mathrm{ADC}$ set calculation. Both readers reported that the reproducibility of the ADC measurements for f-ADC, z-ADC, sADCb1000, and s-ADCb1500 was excellent for all the tissues, while the reliability of the $\mathrm{ADC}$ measurements for $\mathrm{s}-\mathrm{ADC}_{\mathrm{b} 50}$ was good. The inter-reader consistency of all the ADC set measurements was excellent for all the tissues. Table 4 shows the consistency of the ADC values between the $\mathrm{z}-\mathrm{ADC}$ and s-ADC sets. The consistency of the ADC values in the transitional zone between $\mathrm{z}$-DWI and s-DWI $\mathrm{b}_{\mathrm{b} 50}$ was good, and the consistency of the $\mathrm{ADC}$ values between $\mathrm{z}-\mathrm{ADC}$ and $\mathrm{s}-\mathrm{ADC}_{\mathrm{b} 50}$ for the remaining tissues was excellent. For the $\mathrm{s}-\mathrm{ADC}_{\mathrm{b} 1000}$ and $\mathrm{s}-\mathrm{ADC}_{\mathrm{b} 1500}$ values, the consistency of the ADC values for $\mathrm{z}-\mathrm{ADC}$ for all the tissues was excellent.

\section{Tumor Detection Assessment}

Among the three s-ADC sets, $\mathrm{s}-\mathrm{ADC}_{\mathrm{b} 1000}$ performed the best in the image quality assessment and $\mathrm{ADC}$ evaluation. Therefore, it was selected for further comparisons with $\mathrm{f}-\mathrm{ADC}$ and $\mathrm{z}$-ADC in terms of tumor detection and classification (Figure 6). The ADC values for patients with malignant lesions and those with benign lesions measured by the two readers were used to compute the ROC curves (Figure 7). The comparisons of AUCs for both readers based on the $\mathrm{f}-\mathrm{ADC}, \mathrm{z}-\mathrm{ADC}$, and $\mathrm{s}-\mathrm{ADC}$ sets are summarized in Table 5. Both the $\mathrm{z}-\mathrm{ADC}$ and s-ADC sets showed significantly better predictive capabilities than the $\mathrm{f}$-ADC set $(P \leq 0.027)$. The differences in AUCs between s$\mathrm{ADC}$ and $\mathrm{z}-\mathrm{ADC}$ were not statistically significant (reader 1: $z=$ $0.134, P=0.893$; reader $2: z=0.094, P=0.925)$. 
TABLE 3 | The mean apparent diffusion coefficient (ADC) values $\left(\times 10^{-3} \mathrm{~mm}^{2} / \mathrm{s}\right)$ of the different ADC sets.

\begin{tabular}{|c|c|c|c|c|}
\hline \multirow[t]{2}{*}{ Parameter } & \multicolumn{4}{|c|}{ ADC value $\left(\times 10^{-3} \mathrm{~mm}^{2} / \mathrm{s}\right)$} \\
\hline & Peripheral zone $(n=10)$ & Transitional zone $(n=10)$ & Benign lesions $(n=26)$ & Malignant lesions $(n=24)$ \\
\hline \multicolumn{5}{|l|}{ Reader 1} \\
\hline$f-A D C$ & $1.90 \pm 0.11$ & $1.41 \pm 0.13$ & $1.40 \pm 0.28$ & $1.06 \pm 0.25$ \\
\hline Z-ADC & $1.43 \pm 0.17$ & $1.20 \pm 0.16$ & $0.98 \pm 0.18$ & $0.61 \pm 0.11$ \\
\hline $\mathrm{s}-\mathrm{ADC}_{\mathrm{b} 50}$ & $1.43 \pm 0.25$ & $1.20 \pm 0.18$ & $1.09 \pm 0.23$ & $0.68 \pm 0.13$ \\
\hline$s-A D C_{b 1000}$ & $1.43 \pm 0.16$ & $1.20 \pm 0.16$ & $0.99 \pm 0.18$ & $0.61 \pm 0.17$ \\
\hline$s-A D C_{b 1500}$ & $1.46 \pm 0.18$ & $1.26 \pm 0.16$ & $1.01 \pm 0.17$ & $0.67 \pm 0.18$ \\
\hline \multicolumn{5}{|l|}{ Reader 2} \\
\hline$f-A D C$ & $1.94 \pm 0.14$ & $1.39 \pm 0.19$ & $1.42 \pm 0.29$ & $1.06 \pm 0.25$ \\
\hline z-ADC & $1.49 \pm 0.16$ & $1.22 \pm 0.14$ & $0.98 \pm 0.18$ & $0.61 \pm 0.11$ \\
\hline $\mathrm{s}-\mathrm{ADC}_{\mathrm{b} 50}$ & $1.44 \pm 0.13$ & $1.18 \pm 0.14$ & $1.02 \pm 0.24$ & $0.69 \pm 0.13$ \\
\hline $\mathrm{s}-\mathrm{ADC}_{\mathrm{b} 1000}$ & $1.48 \pm 0.21$ & $1.18 \pm 0.13$ & $0.99 \pm 0.16$ & $0.61 \pm 0.15$ \\
\hline$s-A_{D C} b_{1500}$ & $1.45 \pm 0.12$ & $1.18 \pm 0.09$ & $1.00 \pm 0.16$ & $0.70 \pm 0.10$ \\
\hline
\end{tabular}

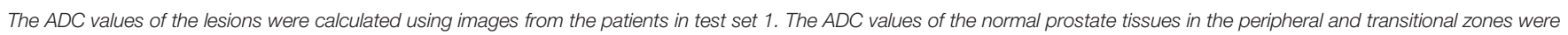
calculated using images from the healthy volunteers in test set 2.

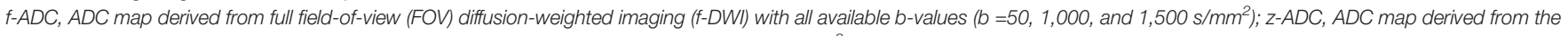

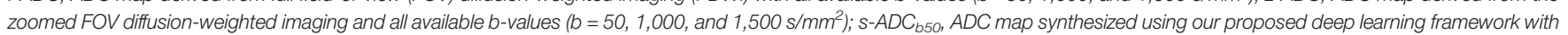

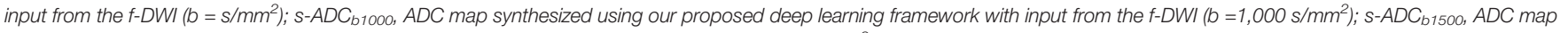
synthesized using our proposed deep learning framework with input from the $f$-DWI $\left(b=1,500 \mathrm{~s} / \mathrm{mm}^{2}\right)$.

\section{DISCUSSION}

The main contribution of our study to the literature is the proposed GAN-based ADC synthesis method that can be used to generate s-ADC maps using single $b$-value DWIs with better image quality and stronger ADC calculation accuracy and reproducibility than a full FOV ADC, but without dependence on software, hardware, and additional scanning time that zoomed FOV ADC technology requires. A shorter scan time will lead to better patient comfort and fewer motion artifacts due to involuntary or autonomous motions. The high reproducibility and accuracy of the ADC calculations may effectively reduce the risk of delayed treatment or unnecessary overtreatment due to the misdiagnoses of benign and malignant lesions. Therefore, the GAN-based ADC synthesis method can increase the clinical benefits to patients, reduce treatment times, and lower the costs incurred by patients and hospitals.

In previous studies (31-33), GANs have been used successfully for image-to-image transformations, such as in generating MRI or PET images using CT images or synthesizing CT images from MRI images, and they have performed well in terms of the traditional pixel-wise metrics. However, GAN-generated images do not have a physical meaning, and they can often lead to spurious images (21). As a result, it is difficult for GANs and their extensions to win the trust of clinicians. Therefore, we not only compared traditional pixelwise metrics, including the RMSE, SSIM, FSIM, and PSNR scores, between the s-ADC sets and reference images, but we also compared the s-ADC and ADC values generated by traditional methods to evaluate the clinical value of GANgenerated images.

In the present study, we evaluated s-ADC maps that were based on DWI inputs with different $b$-values and found that the choice of $b$-values influenced the s-ADC values. Based on a subjective visual evaluation, the $\mathrm{s}-\mathrm{ADC}_{\mathrm{b} 1000}$ maps delineated normal tissues and lesions more clearly than the $\mathrm{s}-\mathrm{ADC}_{\mathrm{b} 50}$ maps, and they provided more details for targeted images than the $\mathrm{s}-\mathrm{ADC}_{\mathrm{b} 1500}$ set. The quantitative evaluation results are also consistent with the visual evaluation results. Among the three sADC sets, the s-ADC $\mathrm{b} 1000$ set achieved a lower RMSE score and higher SSIM, FSIM, and PSNR scores than the s- $\mathrm{ADC}_{\mathrm{b} 50}$ and s$\mathrm{ADC}_{\mathrm{b} 1500}$ sets, indicating that the $\mathrm{s}-\mathrm{ADC}_{\mathrm{b} 1000}$ set is more similar to the realistic $\mathrm{z}$-ADC in terms of noise distribution, image structure, and features. Additionally, the s- $\mathrm{ADC}_{\mathrm{b} 1000}$ set showed better intra-reader repeatability and inter-reader consistency than the $\mathrm{s}-\mathrm{ADC}_{\mathrm{b} 50}$ and $\mathrm{s}-\mathrm{ADC}_{\mathrm{b} 1500}$ sets. Moreover, the $\mathrm{s}$ $\mathrm{ADC}_{\mathrm{b} 1000}$ set showed the best $\mathrm{ADC}$ value inter-method consistency with the $\mathrm{z}$-ADC set, suggesting that a DWI with a $b$-value of $1,000 \mathrm{~s} / \mathrm{mm}^{2}$ might be more suitable for synthesizing ADC maps than one with a $b$-value of 50 or $1,500 \mathrm{~s} / \mathrm{mm}^{2}$. The similarity between the target image z-DWI and s-DWI strongly depends on how much useful information the input f-DWI can provide to the generator for the extraction of meaningful features to begin the mapping between f-DWI and z-ADC. Low- $b$-value DWIs suffer from T2 shine-through or black-through effects, whereas high- $b$-value DWIs might be affected by diffusion kurtosis effects (34). These effects have a negative influence on image quality and lesion information, causing a relatively lower similarity between the $\mathrm{s}-\mathrm{ADC}_{\mathrm{b} 50}$ and $\mathrm{s}-\mathrm{ADC}_{\mathrm{b} 1500}$ sets and the $\mathrm{z}$ $\mathrm{ADC}$ set compared to the $\mathrm{s}-\mathrm{ADC}_{\mathrm{b} 1000}$ set (3).

In our study, both the z-ADC and s-ADC sets showed less distortion and better reproducibility of the quantitative ADC values for all the evaluated tissues; they also showed better tumor detection and classification capacity than the f-ADC sets. The $\mathrm{ADC}$ values are generated for most of the current clinical implementations by calculating the signal intensity decay using two or more DWI sets with different $b$-values $(1-5,9-11,13,14)$. The reproducibility and accuracy of the calculated ADC values are affected by the choice of $b$-values $(3,4,34)$ and the DWI image quality (14). The application of a significant number of 


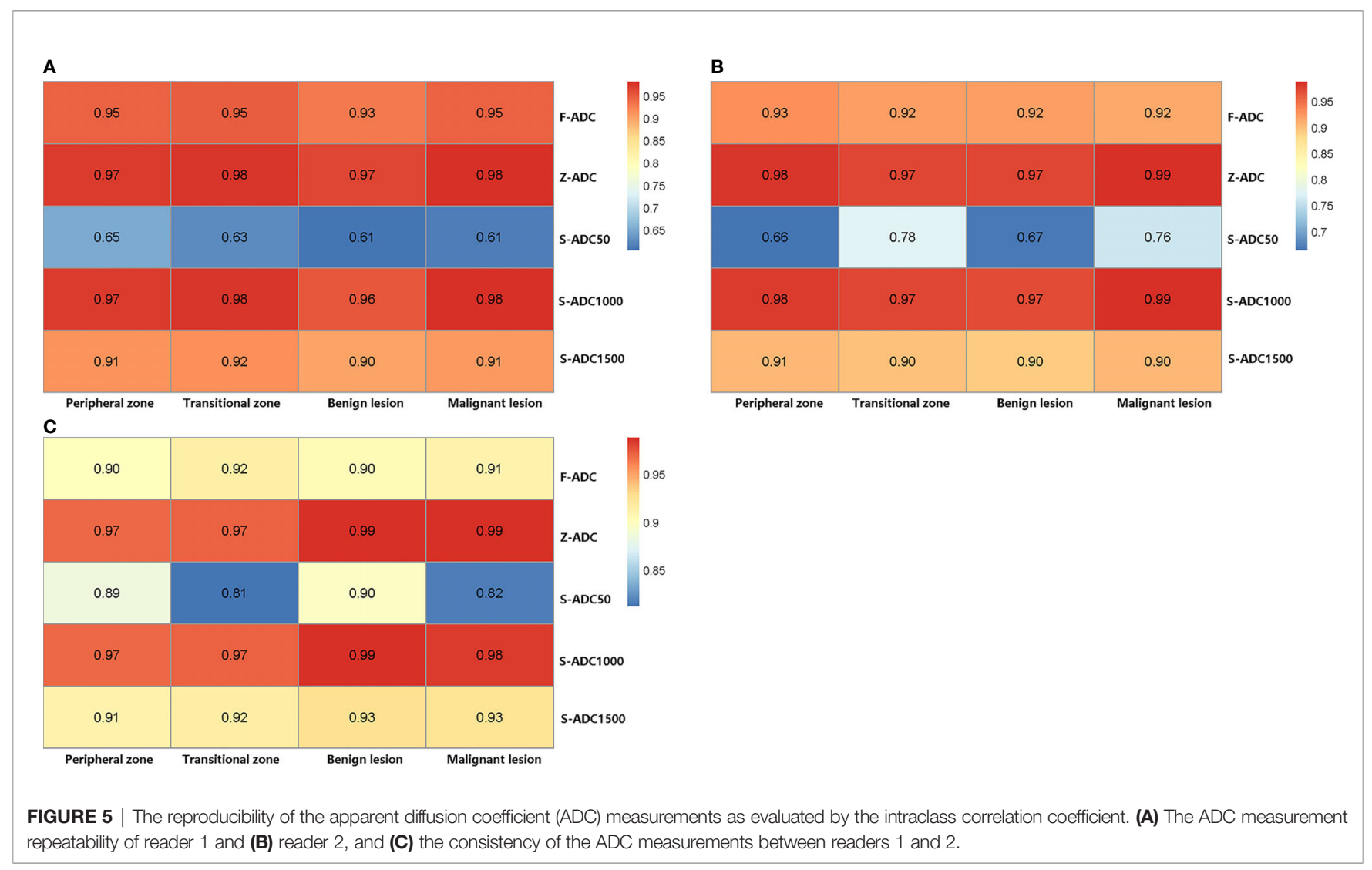

TABLE 4 | Comparison between the inter-method intraclass correlation coefficients from the z-DWI and s-DWI sets.

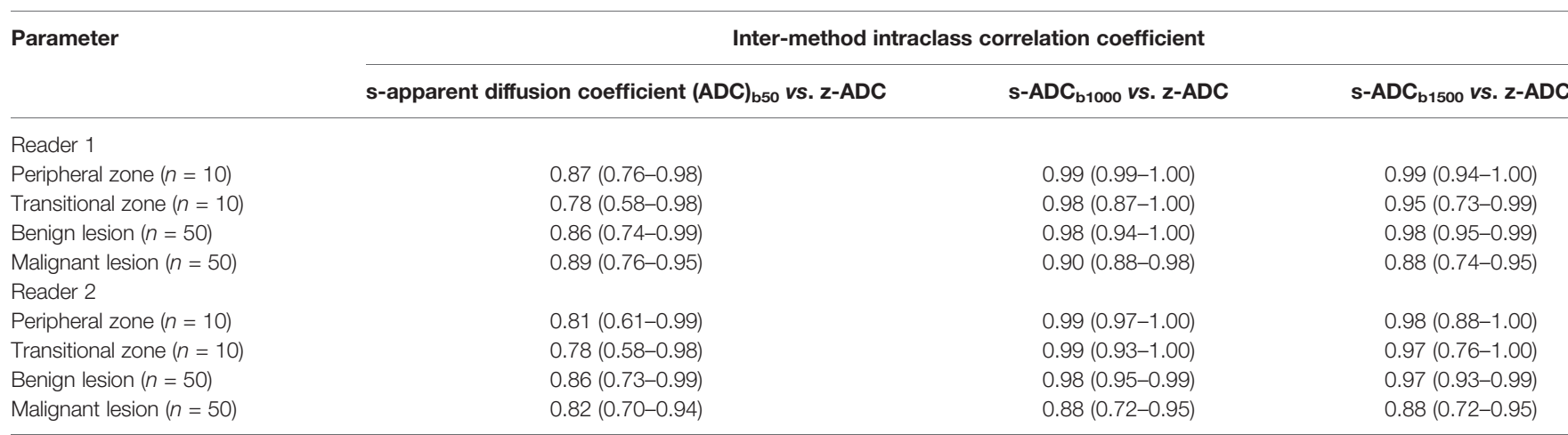

z-ADC, ADC map derived from zoomed field-of view (FOV) diffusion-weighted imaging and all the available $b$-values $\left(b=50,1,000, \text { and 1,500 } s / m^{2}\right)^{2} ; s-A D C_{b 50}, A D C$ map synthesized using our proposed deep learning framework with input from full FOV diffusion-weighted imaging (f-DWI) $\left(b=50 \mathrm{~s} / \mathrm{mm}^{2}\right)$; $s-A D C_{b 1000}$, $A D C$ map synthesized using our proposed deep learning framework with input from $\mathrm{f}$-DWI $\left(b=1,000 \mathrm{~s} / \mathrm{mm}^{2}\right)$; $s-A D C_{b 1500}, A D C$ map synthesized using our proposed deep learning framework with input from $f$-DWI $\left(b=1500 \mathrm{~s} / \mathrm{mm}^{2}\right)$.

$b$-values improves the reproducibility and accuracy of the calculated ADC values, although it also increases the scanning time $(3,35)$. In contrast to traditional ADC calculation methods, our proposed method takes advantage of the ability of GAN to simulate data distribution and synthesize ADC maps that are highly similar to real zoomed FOV ADC maps that use a full FOV DWI with a single $b$-value. Considering the excellent image quality consistency and similar tumor detection and classification abilities between the s-ADC and z-ADC maps, we believe that the deep learning algorithm might be a feasible method for generating ADC maps as an alternative to $\mathrm{z}$-ADC maps without requiring a strong dependence on software, hardware, and additional scan time (36).

Our study has several limitations. First, the s-ADC $\mathrm{b}_{\mathrm{b} 1000}$ set showed the best image quality among the s-ADC sets; however, it remains unknown whether a DWI set with a $b$-value of 1,000 


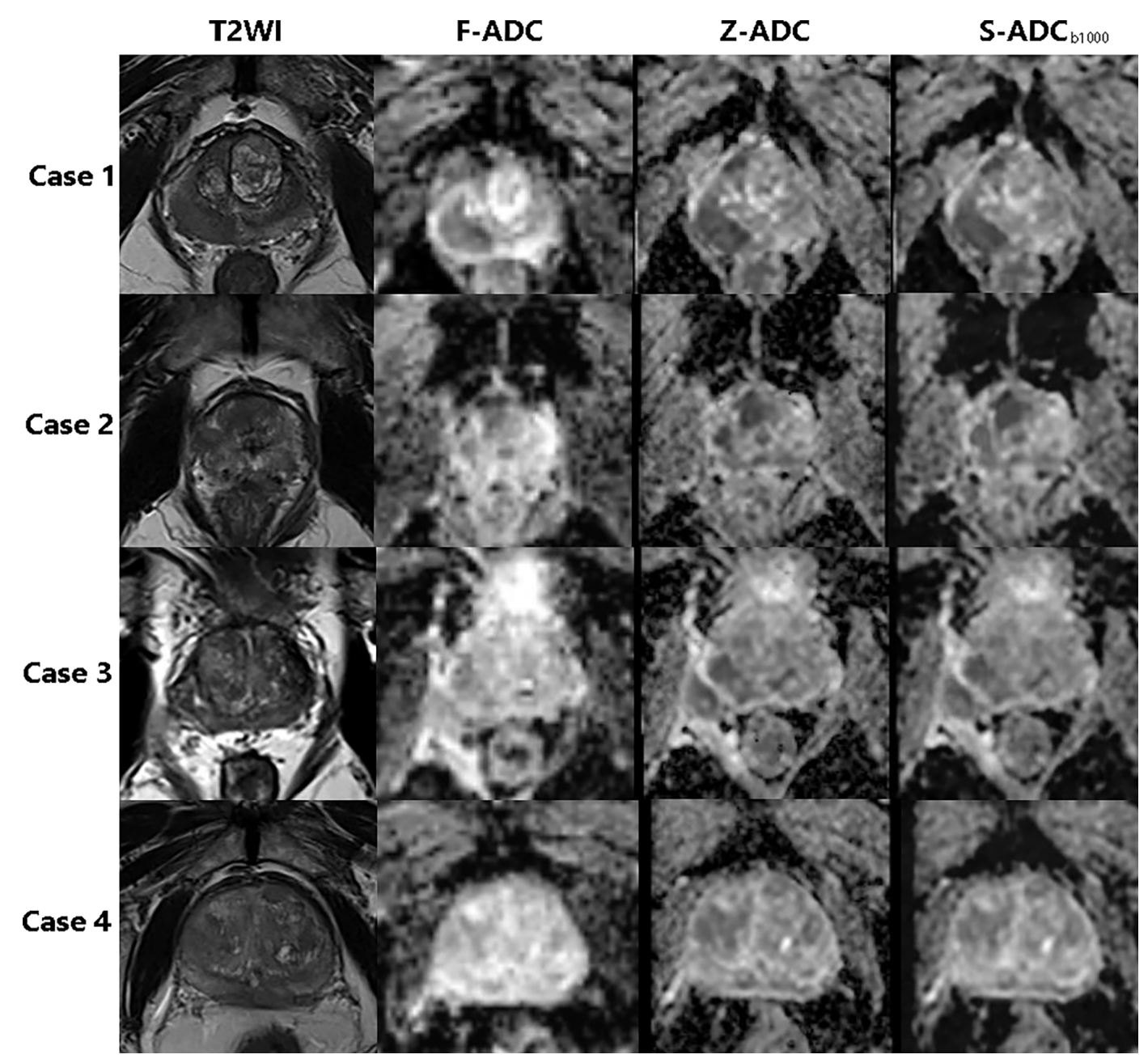

FIGURE 6 | The T2-weighted image, f-ADC, z-ADC, and s-ADC $\mathrm{b}_{\mathrm{b} 1000}$ of four different patients. Case 1: An 85-year-old man with prostate cancer in the right peripheral zone and an initial prostate-specific antigen level of $0.157 \mathrm{ng} / \mathrm{ml}$. Case 2: An 85-year-old man with prostate cancer in the central zone and an initial prostate-specific antigen level of $21.44 \mathrm{ng} / \mathrm{ml}$. Case 3: A 67-year-old man with an inflammatory nodule in the right peripheral zone and an initial prostate-specific antigen level of $14.37 \mathrm{ng} / \mathrm{ml}$. Case 4: A 77 -year-old man with prostate cancer in the central zone and an initial prostate-specific antigen level of 56.62 ng/ml.
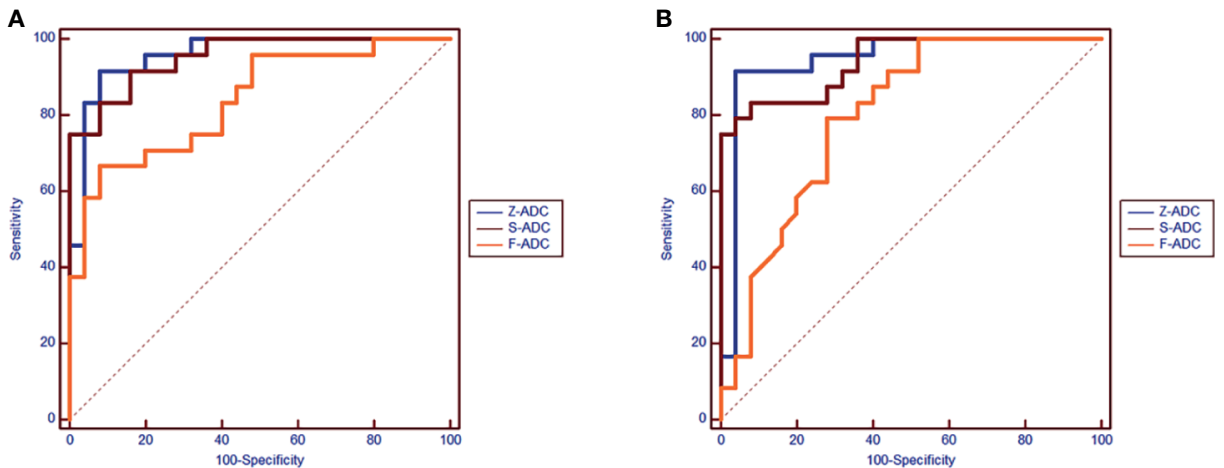

FIGURE 7 | The receiver operating characteristic comparison of the diagnostic accuracy of the f-ADC, z-ADC, and s-ADCb1000 sets (A: reader 1, B: reader 2). 
TABLE 5 | The comparison of areas under the curve (AUCs) based on the f-apparent diffusion coefficient (ADC), z-ADC, and s-ADC sets for both readers.

\begin{tabular}{|c|c|c|c|c|}
\hline & & z-ADC vs. s-ADC $\mathrm{b} 1000$ & z-ADC vs. f-ADC & s-ADC ${ }_{\text {b1000 }}$ vs. f-ADC \\
\hline \multirow[t]{3}{*}{ Reader-1 } & AUC & 0.96 vs. 0.95 & 0.96 vs. 0.84 & 0.95 vs. 0.84 \\
\hline & $z$ value & 0.134 & 2.445 & 2.207 \\
\hline & $P$-value & 0.893 & 0.015 & 0.027 \\
\hline \multirow[t]{3}{*}{ Reader-2 } & AUC & 0.94 vs. 0.94 & 0.94 vs. 0.80 & 0.94 vs. 0.80 \\
\hline & $z$ value & 0.094 & 2.652 & 2.29 \\
\hline & $P$-value & 0.925 & 0.008 & 0.022 \\
\hline
\end{tabular}

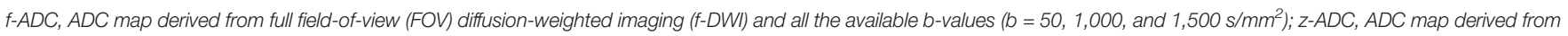

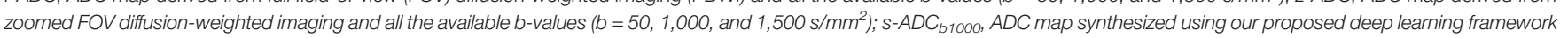
with input from $\mathrm{f}-\mathrm{DWI}\left(\mathrm{b}=1,000 \mathrm{~s} / \mathrm{mm}^{2}\right)$.

$\mathrm{s} / \mathrm{mm}^{2}$ is the most appropriate for ADC map synthesis. In future studies, s-ADC sets generated using DWI sets with more potential $b$-values should be compared. Second, as ADC values vary across vendors, the generalizability of our model across MRI scanners from different vendors requires multi-center verification.

In conclusion, the GAN-based ADC synthesis method can generate s-ADC maps using a single $b$-value DWI with good image quality and high reproducibility and ADC calculation accuracy.

\section{DATA AVAILABILITY STATEMENT}

The original contributions presented in the study are included in the article/supplementary material. Further inquiries can be directed to the corresponding author.

\section{ETHICS STATEMENT}

The studies involving human participants were reviewed and approved by The ethics committee of Shanghai Jiao Tong University Affiliated Sixth People's Hospital. The patients/ participants provided their written informed consent to participate in this study. Written informed consent was

\section{REFERENCES}

1. Turkbey B, Shah VP, Pang Y, Bernardo M, Xu S, Kruecker J, et al. Is Apparent Diffusion Coefficient Associated With Clinical Risk Scores for Prostate Cancers That are Visible on 3-T MR Images? Radiology (2011) 258(2):48895. doi: 10.1148/radiol.10100667

2. Hambrock T, Somford DM, Huisman HJ, van Oort IM, Witjes JA, Hulsbergen-van de Kaa CA, et al. Relationship Between Apparent Diffusion Coefficients at 3.0-T MR Imaging and Gleason Grade in Peripheral Zone Prostate Cancer. Radiology (2011) 259(2):453-61. doi: 10.1148/radiol.11091409

3. Turkbey B, Rosenkrantz AB, Haider MA, Padhani AR, Villeirs G, Macura KJ, et al. Prostate Imaging Reporting and Data System Version 2.1: 2019 Update of Prostate Imaging Reporting and Data System Version 2. Eur Urol (2019) 76 (3):340-51. doi: 10.1016/j.eururo.2019.02.033

4. Surov A, Meyer HJ, Wienke A. Correlations Between Apparent Diffusion Coefficient and Gleason Score in Prostate Cancer: A Systematic Review. Eur Urol Oncol (2020) 3(4):489-97. doi: 10.1016/j.euo.2018.12.006

5. Bai K, Sun Y, Li W, Zhang L. Apparent Diffusion Coefficient in Extraprostatic Extension of Prostate Cancer: A Systematic Review and Diagnostic MetaAnalysis. Cancer Manag Res (2019) 11:3125-37. doi: 10.2147/CMAR.S191738 obtained from the individual(s) for the publication of any potentially identifiable images or data included in this article.

\section{AUTHOR CONTRIBUTIONS}

JZ is the guarantor of the integrity of the entire study (study concepts/study design, data acquisition, and data analysis/ interpretation). LH participated in statistical analysis, manuscript drafting and manuscript revision for important intellectual content. $\mathrm{LH}$ and $\mathrm{JZ}$ edited the manuscript. $\mathrm{LH}$ and $\mathrm{DZ}$ participated in literature research. LH, DZ, YX, and LW participated in clinical studies. All authors contributed to the article and approved the submitted version.

\section{FUNDING}

This study was funded by the National Natural Science Foundation of China (nos. 81901845 and 81671791), the Science Foundation of Shanghai Jiao Tong University Affiliated Sixth People's Hospital (no. 201818), and the Shanghai Key Discipline of Medical Imaging (no. 2017ZZ02005).

6. Nguyen TB, Ushinsky A, Yang A, Nguyentat M, Fardin S, Uchio E, et al Utility of Quantitative Apparent Diffusion Coefficient Measurements and Normalized Apparent Diffusion Coefficient Ratios in the Diagnosis of Clinically Significant Peripheral Zone Prostate Cancer. Br J Radiol (2018) 91(1088):20180091. doi: 10.1259/bjr.20180091

7. Kato S, Kamijima S, Inaoka T, Kamiya N, Sasai D, Terada H, et al. Quantitative Evaluation of the Relative Apparent Diffusion Coefficient Values on Multiparametric Magnetic Resonance Imaging to Predict Higher Gleason Score Prostate Cancer. Scand J Urol (2018) 52(3):180-5. doi: 10.1080/ 21681805.2018.1481143

8. Nougaret S, Robertson N, Golia Pernicka J, Molinari N, Hötker AM, Ehdaie B, et al. The Performance of PI-RADSv2 and Quantitative Apparent Diffusion Coefficient for Predicting Confirmatory Prostate Biopsy Findings in Patients Considered for Active Surveillance of Prostate Cancer. Abdom Radiol (NY) (2017) 42(7):1968-74. doi: 10.1007/s00261-017-1086-7

9. Gupta RT, Kauffman CR, Garcia-Reyes K, Palmeri ML, Madden JF, Polascik TJ, et al. Apparent Diffusion Coefficient Values of the Benign Central Zone of the Prostate: Comparison With Low- and High-Grade Prostate Cancer. AJR Am J Roentgenol (2015) 205(2):331-6. doi: 10.2214/AJR.14.14221

10. Boesen L, Chabanova E, Løgager V, Balslev I, Thomsen HS. Apparent Diffusion Coefficient Ratio Correlates Significantly With Prostate Cancer 
Gleason Score at Final Pathology. J Magn Reson Imaging (2015) 42(2):446-53. doi: 10.1002/jmri.24801

11. Babourina-Brooks B, Cowin GJ, Wang D. Diffusion-Weighted Imaging in the Prostate: An Apparent Diffusion Coefficient Comparison of Half-Fourier Acquisition Single-Shot Turbo Spin-Echo and Echo Planar Imaging. Magn Reson Imaging (2012) 30(2):189-94. doi: 10.1016/j.mri.2011.09.024

12. Nketiah G, Selnaes KM, Sandsmark E, Teruel JR, Krüger-Stokke B, Bertilsson $\mathrm{H}$, et al. Geometric Distortion Correction in Prostate Diffusion-Weighted MRI and its Effect on Quantitative Apparent Diffusion Coefficient Analysis. Magn Reson Med (2018) 79(5):2524-32. doi: 10.1002/mrm.26899

13. Mazaheri Y, Vargas HA, Nyman G, Shukla-Dave A, Akin O, Hricak H. DiffusionWeighted MRI of the Prostate at 3.0 T: Comparison of Endorectal Coil (ERC) MRI and Phased-Array Coil (PAC) MRI-The Impact of SNR on ADC Measurement. Eur J Radiol (2013) 82(10):e515-20. doi: 10.1016/j.ejrad.2013.04.041

14. Gibbs P, Pickles MD, Turnbull LW. Repeatability of Echo-Planar-Based Diffusion Measurements of the Human Prostate at 3 T. Magn Reson Imaging (2007) 25(10):1423-9. doi: 10.1016/j.mri.2007.03.030

15. Henkelman RM. Measurement of Signal Intensities in the Presence of Noise in MR Images. Med Phys (1985) 12(2):232-33. doi: 10.1118/1.595711

16. Gudbjartsson H, Patz S. The Rician Distribution of Noisy MRI Data. Magn Reson Med (1995) 34(6):910-14. doi: 10.1002/mrm.1910340618

17. Riffel P, Michaely HJ, Morelli JN, Pfeuffer J, Attenberger UI, Schoenberg SO, et al. Zoomed EPI-DWI of the Pancreas Using Two-Dimensional Spatially Selective Radiofrequency Excitation Pulses. PloS One (2014) 9(3):e89468. doi: 10.1371/journal.pone.0089468

18. Rosenkrantz AB, Chandarana H, Pfeuffer J, Triolo MJ, Shaikh MB, Mossa DJ, et al. Zoomed Echo-Planar Imaging Using Parallel Transmission: Impact on Image Quality of Diffusion-Weighted Imaging of the Prostate at 3T. Abdom Imaging (2015) 40(1):120-26. doi: 10.1007/s00261-014-0181-2

19. Thierfelder KM, Scherr MK, Notohamiprodjo M, Weiß J, Dietrich O, MuellerLisse UG, et al. Diffusion-Weighted MRI of the Prostate: Advantages of Zoomed EPI With Parallel-Transmit-Accelerated 2D-Selective Excitation Imaging. Eur Radiol (2014) 24(12):3233-41. doi: 10.1007/s00330-014-3347-y

20. Goodfellow I, Pouget-Abadie J, Mirza M, Xu B, Warde-Farley D, Ozair S, et al. Generative Adversarial Nets. In: Advances in Neural Information Processing Systems. The MIT Press (2014)p. 2672-80.

21. Armanious K, Jiang C, Fischer M, Kustner T, Hepp T, Nikolaou K, et al. MedGAN: Medical Image Translation Using GANs. Comput Med Imaging Graph (2020) 79:101684. doi: 10.1016/j.compmedimag.2019.101684

22. Wolterink JM, Leiner T, Viergever MA, Isgum I. Generative Adversarial Networks for Noise Reduction in Low-Dose CT. IEEE Trans Med Imaging (2017) 36(12):2536-45. doi: 10.1109/TMI.2017.2708987

23. Kim KH, Do WJ, Park SH. Improving Resolution of MR Images With an Adversarial Network Incorporating Images With Different Contrast. Med Phys (2018) 45(7):3120-31. doi: 10.1002/mp.12945

24. Wang J, Zhao Y, Noble JH, Dawant BM. Conditional Generative Adversarial Networks for Metal Artifact Reduction in CT Images of the Ear. Med Image Comput Dawna Assist Interv (2018) 11070:3-11. doi: 10.1007/978-3-030-00928-1_1

25. Johnson PM, Drangova M. Conditional Generative Adversarial Network for 3D Rigid-Body Motion Correction in MRI. Magn Reson Med (2019) 82 (3):901-10. doi: 10.1002/mrm.27772

26. Cohen JP, Luck M, Honari S. (2018). Distribution Matching Losses can Hallucinate Features in Medical Image Translation, in: International Conference on Medical Image Computing and Computer-Assisted Intervention,
Medical Image Computing and Computer Assisted Intervention - MICCAI 2018, Lecture Notes in Computer Science, pp. 529-36.

27. Xu H, Huang C, Huang X, Xu C, Huang M. Combining Convolutional Neural Network Markov Random Field Semantic Image Retrieval. Adv Multimedia (2018) 2018:1-11.

28. Simonyan K, Zisserman A. Very Deep Convolutional Networksfor Large-Scale Image Recognition. The 3rd International Conference on Learning Representations (2014).

29. Chen C, Liu W, Tan X, Wong KK. Semi-Supervised Learning for Face Sketch Synthesis in the Wild. In: C Jawahar, $\mathrm{H} \mathrm{Li}, \mathrm{G}$ Mori, K Schindler, editors. Computer Vision - ACCV 2018. Lecture Notes in Computer Science, vol. 11361. Cham: Springer (2018). doi: 10.1007/978-3-030-20887-5_14

30. Zhou F, Yao R, Liu B, Qiu G. Visual Quality Assessment for Super-Resolved Images: Database and Method. IEEE Trans Image Process (2019) 28(7):352841. doi: 10.1109/TIP.2019.2898638

31. Jin CB, Kim H, Liu M, Jung W, Joo S, Park E, et al. Deep CT to MR Synthesis Using Paired and Unpaired Data. Sensors (Basel) (2019) 19(10):2361. doi: 10.3390/s19102361

32. Nie D, Trullo R, Lian J, Petitjean C, Ruan S, Wang Q, et al. Medical Image Synthesis With Context-Aware Generative Adversarial Networks. Med Image Comput Jinn Assist Interv (2017) 10435:417-25. doi: 10.1007/978-3-31966179-7_48

33. Bentaieb A, Hamarneh GJI. ToMI. Adversarial Stain Transf Histopathol Image Anal (2017) PP(99):1-1.

34. Peng Y, Jiang Y, Antic T, Sethi I, Schmid-Tannwald C, Eggener S, et al. Apparent Diffusion Coefficient for Prostate Cancer Imaging: Impact of B Values. AJR Am J Roentgenol (2014) 202(3):W247-53. doi: 10.2214/AJR.13.10917

35. Park SY, Kim CK, Park BK, Kwon GY. Comparison of Apparent Diffusion Coefficient Calculation Between Two-Point and Multipoint B Value Analyses in Prostate Cancer and Benign Prostate Tissue at 3 T: Preliminary Experience. AJR Am J Roentgenol (2014) 203(3):W287-94. doi: 10.2214/AJR.13.11818

36. Hu L, Zhou DW, Fu CX, Benkert T, Jiang CY, Li RT, et al. Advanced Zoomed Diffusion-Weighted Imaging vs. Full-Field-of-View Diffusion-Weighted Imaging in Prostate Cancer Detection: A Radiomic Features Study. Eur Radiol (2021) 31(3):1760-9. doi: 10.1007/s00330-020-07227-4

Conflict of Interest: CF was employed by Siemens Shenzhen Magnetic Resonance, Ltd. TB was employed by MR Application Predevelopment, Siemens Healthcare GmbH, Erlangen, Germany.

The remaining authors declare that the research was conducted in the absence of any commercial or financial relationships that could be construed as a potential conflict of interest.

Publisher's Note: All claims expressed in this article are solely those of the authors and do not necessarily represent those of their affiliated organizations, or those of the publisher, the editors and the reviewers. Any product that may be evaluated in this article, or claim that may be made by its manufacturer, is not guaranteed or endorsed by the publisher.

Copyright $\odot 2021 \mathrm{Hu}$, Zhou, Fu, Benkert, Xiao, Wei and Zhao. This is an openaccess article distributed under the terms of the Creative Commons Attribution License (CC BY). The use, distribution or reproduction in other forums is permitted, provided the original author(s) and the copyright owner(s) are credited and that the original publication in this journal is cited, in accordance with accepted academic practice. No use, distribution or reproduction is permitted which does not comply with these terms. 